\title{
Bridging the Gap Among Clinical Practice Guidelines for Pain Management in Cancer and Sickle Cell Disease
}

\author{
Alyssa A. Schatz, MSW'i ; Thomas K. Oliver, BAㄹ; Robert A. Swarm, MD1,3; Judith A. Paice, PhD, RN ${ }^{1,2,4}$; \\ Deepika S. Darbari, MD 5,6; Deborah Dowell, MD7; Salimah H. Meghani, $\mathrm{PhD}^{8}$; Katy Winckworth-Prejsnar, $\mathrm{MPH}^{1}$; \\ Eduardo Bruera, MD², ; Robert M. Plovnick, MD, MS5; Lisa Richardson, MD7; Neha Vapiwala, MD; \\ Dana Wollins, MGC, DrPH²; Clifford A. Hudis, MD²; and Robert W. Carlson, MD ${ }^{1}$
}

\begin{abstract}
Opioids are a critical component of pain relief strategies for the management of patients with cancer and sickle cell disease. The escalation of opioid addiction and overdose in the United States has led to increased scrutiny of opioid prescribing practices. Multiple reports have revealed that regulatory and coverage policies, intended to curb inappropriate opioid use, have created significant barriers for many patients. The Centers for Disease Control and Prevention, National Comprehensive Cancer Network, and American Society of Clinical Oncology each publish clinical practice guidelines for the management of chronic pain. A recent JAMA Oncology article highlighted perceived variability in recommendations among these guidelines. In response, leadership from guideline organizations, government representatives, and authors of the original article met to discuss challenges and solutions. The meeting featured remarks by the Commissioner of Food and Drugs, presentations on each clinical practice guideline, an overview of the pain management needs of patients with sickle cell disease, an overview of perceived differences among guidelines, and a discussion of differences and commonalities among the guidelines. The meeting revealed that although each guideline varies in the intended patient population, target audience, and methodology, there is no disagreement among recommendations when applied to the appropriate patient and clinical situation. It was determined that clarification and education are needed regarding the intent, patient population, and scope of each clinical practice guideline, rather than harmonization of guideline recommendations. Clinical practice guidelines can serve as a resource for policymakers and payers to inform policy and coverage determinations.
\end{abstract}

J Natl Compr Canc Netw 2020;18(4):392-399 doi: 10.6004/jnccn.2019.7379

\footnotetext{
${ }^{1}$ National Comprehensive Cancer Network, Plymouth Meeting, Pennsylvania; ${ }^{2}$ American Society of Clinical Oncology, Alexandria, Virginia; ${ }^{3}$ Washington University School of Medicine, St. Louis, Missouri; ${ }^{4}$ Northwestern University, Feinberg School of Medicine, Chicago, Illinois; ${ }^{5}$ American Society of Hematology, Washington, DC; ${ }^{6}$ Children's National Medical Center, George Washington University School of Medicine and Health Sciences, Washington, DC; ${ }^{7}$ Centers for Disease Control and Prevention, Atlanta, Georgia; ${ }^{8}$ University of Pennsylvania, Philadelphia, Pennsylvania; and ${ }^{9}$ University of Texas MD Anderson Cancer Center, Houston, Texas.
}

Opioids are an established and effective tool in the armamentarium of pain relief strategies for the management of complex, acute, and/or persistent pain in select disease-specific patient populations. However, in recent years the escalation of opioid addiction and overdose in the United States has led to increased scrutiny of opioid prescribing practices. In the management of cancer and sickle cell disease, multiple reports indicate that a patchwork of regulatory and coverage policies, intended to curb inappropriate opioid use, has led to significant barriers for many cancer patients when opioid use is clinically indicated for pain management. ${ }^{1,2}$

Pain management is complex and multifactorial. Clinical practice guidelines from leading organizations play a crucial role in informing appropriate opioid prescribing practices for patients in general and for select groups with unique pain management needs. A recent JAMA Oncology article ${ }^{3}$ highlighted perceived variability in the scope and recommendations among commonly used clinical practice guidelines for cancer-related pain. The article referenced guidelines from the Centers for Disease Control and Prevention (CDC), ${ }^{4}$ NCCN, ${ }^{5,6}$ and ASCO. ${ }^{7}$ The authors of that article contend that variability in clinical practice guidelines may lead to confusion among primary care, oncology, and other prescribers, and highlight the need for collaboration among guideline developers to resolve inconsistencies in guideline recommendations. In response to these concerns, NCCN and ASCO convened a meeting to address perceived variance among clinical practice guidelines for pain management in patients with cancer or sickle cell disease. ${ }^{6}$

In November 2018, NCCN and ASCO held a meeting entitled "Bridging the Critical Divide Among Clinical Practice Guidelines for Cancer Pain and Sickle Cell Disease." Featured attendees included representatives from NCCN, ASCO, the American Society of Hematology (ASH), the CDC, the FDA, and the authors of the JAMA Oncology article. This article outlines the discussion and conclusions from this meeting, including possible 
next steps identified by participants to ensure access to appropriate pain management tools while also minimizing the risk of opioid use disorder for patients with cancer or sickle cell disease. ${ }^{8}$

\section{Opioid-Containing Recommendations in Guidelines for Cancer Pain and Sickle Cell Disease}

\section{The FDA View}

The FDA plays an important role in setting a national regulatory agenda to address opioid misuse and appropriate prescribing practices. Scott Gottlieb, the 23rd FDA Commissioner, addressed the attendees of the meeting and highlighted current FDA initiatives that impact opioid prescribing practices, including collaborations to develop evidence-based opioid prescribing guidelines based on specific clinical indications, as well as changes to the way FDA approves new opioid drugs. ${ }^{8}$

Attempts to address the opioid epidemic have led several states to begin imposing limits on the number of days an opioid prescription can be written for the first fill, with several states instituting 7-day limits. Commissioner Gottlieb noted that, while well intended, these blanket dosing limits fail to consider specific clinical situations in which the appropriate duration of use may be even shorter than 7 days. As such, Commissioner Gottlieb called for science-based prescribing criteria that more closely tie duration to the clinical purpose of the prescription.

Commissioner Gottlieb discussed the FDA's collaboration with the National Academies of Sciences, Engineering, and Medicine to create a scientific framework for developing evidence-based guidelines by indication. The partnership is focused on advancing the development of evidence-based guidelines for appropriate opioid analgesic prescribing for acute pain. In addition to this partnership, Commissioner Gottlieb noted that the FDA is working on an agreement with an academic institution to systematically review data that have been submitted to the FDA as part of new drug applications. The goal is to assess whether the FDA has sufficient data on hand to develop guidelines that would govern prescribing for some common, acute pain indications. In his remarks, Commissioner Gottlieb noted his support for evidencebased guidelines as a tool to differentiate between clinical situations in which opioids are overprescribed and conditions for which opioids may be the only appropriate pain management tool. ${ }^{8}$

\section{Guidelines for the Management of Pain}

CDC, NCCN, ASCO and other groups publish clinical practice guidelines for the management of chronic pain that are widely used by clinicians and other prescribing professionals, patients, and payers to delineate appropriate medical decision-making. These guidelines are intended for varying populations and are developed using differing methodologies. As a result, the recommendations can appear to not be fully aligned, which may lead to confusion among prescribers, payers, patients, and policymakers. The meeting featured expert presentations on each guideline, a presentation on the pain management needs of patients with sickle cell disease, and a subsequent discussion of differences and commonalities among the guidelines (Figure 1 , Table 1). Following is a summary of information reviewed and subsequent discussion at the meeting. Specifically, the proceeding discussion of guidelines provides an overview of each guideline's title, year published, patient population, purpose, target audience, authorship/ expert representation, methodology and evidence source, and feedback processes.

\section{CDC Guideline for Prescribing Opioids for Chronic Pain}

Published in March 2016, the CDC Guideline for Prescribing Opioids for Chronic Pain is intended to ensure patients have access to safer, more effective chronic pain treatment while reducing the number of people who misuse, abuse, or overdose from these drugs. ${ }^{4}$ The CDC Guideline provides recommendations for the prescribing of opioid pain medication for patients aged $\geq 18$ years in primary care settings. Recommendations focus on the use of opioids in treating chronic pain (pain lasting $>3$ months or past the time of normal tissue healing) outside of active cancer treatment, palliative care, and end-oflife care. The target audience for the CDC Guideline is primary care practitioners, including family physicians and internists. CDC developed the guideline using the Grading of Recommendations Assessment, Development, and Evaluation (GRADE) framework, ${ }^{9}$ and recommendations are made based on a systematic review of the scientific evidence while considering benefits and harms, patient and provider values and preferences, and resource allocation. The CDC Guideline was developed with support of a Core Expert Group, which included individual input from subject matter experts in opioid prescribing, substance use disorder treatment, and pain management; representatives of primary care professional societies and state agencies; and an expert in guideline development methodology.

In addition to the Core Expert Group, CDC invited federal partners, including representatives from the Substance Abuse and Mental Health Services Administration, National Institute on Drug Abuse, FDA, US Department of Veterans Affairs, US Department of Defense, Office of the National Coordinator for Health Information Technology, Centers for Medicare and Medicaid Services, Health 


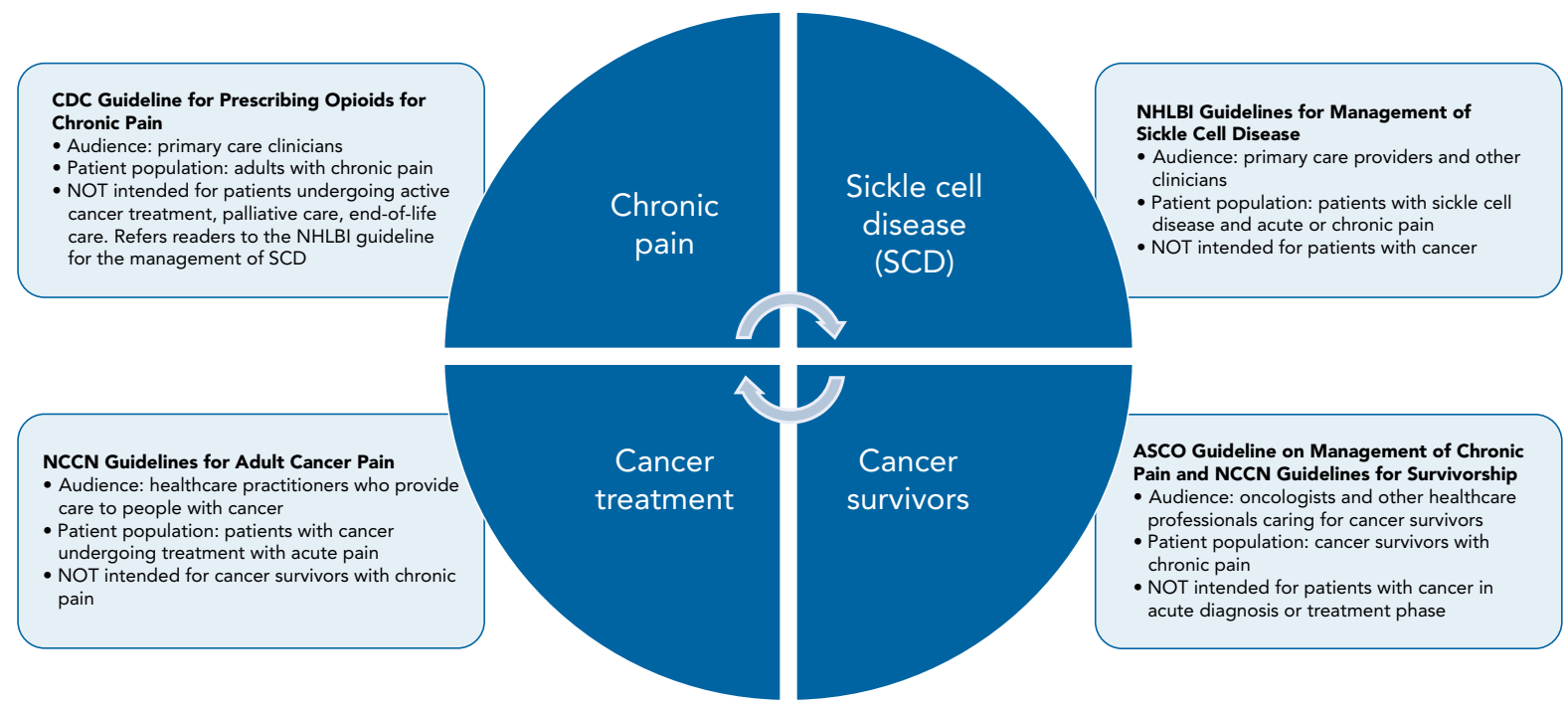

Figure 1. Matrix of clinical practice guidelines for the management of chronic pain. Abbreviation: NHLBI, National Heart, Lung, and Blood Institute.

Resources and Services Administration, Agency for Healthcare Research and Quality, and Office of National Drug Control Policy, to attend the expert meeting and provide feedback on the recommendations. CDC also invited comments from members of a Stakeholder Review Group that included representatives from professional organizations that represent specialties that commonly prescribe opioids (eg, pain medicine, physical medicine and rehabilitation), delivery systems within which opioid prescribing occurs (eg, hospitals), and representation from community organizations with interests in pain management and opioid prescribing. CDC convened a constituent engagement webinar and received comments during and for 2 days following the first webinar. The guideline underwent peer review by 3 experts and public comment was solicited through the Federal Register. Lastly, CDC sought the consensus advice of the National Center for Injury Prevention and Control Board of Scientific Counselors (NCIPC BSC), one of CDC's federal advisory committees. NCIPC BSC established an Opioid Guideline Workgroup to review the quality of the evidence and recommendations, and report their observations to the NCIPC BSC. The workgroup comprised clinicians, subject matter experts, and a patient representative, with the following perspectives represented: primary care, pain medicine, public health, behavioral health, substance abuse treatment, pharmacy, patients, and research. The CDC Guideline was published in the Morbidity and Mortality Weekly Report $(\mathrm{MMWR})^{4}$ and JAMA. ${ }^{10}$

NCCN Guidelines for Adult Cancer Pain

The NCCN Clinical Practice Guidelines in Oncology (NCCN Guidelines) for Adult Cancer Pain identify central principles for the assessment and management of cancer pain in adults, including aspects of opioids, nonopioids, adjuvant analgesics, and broad multimodal care. ${ }^{5}$ The target audience of the NCCN Guidelines for Adult Cancer Pain is healthcare practitioners who provide care to people with cancer, and the patient population covered by these guidelines is adults with cancer-related pain. The NCCN Guidelines for Adult Cancer Pain are authored by a panel of experts representing NCCN Member Institutions, all of which are NCI-designated cancer centers. The NCCN Adult Cancer Pain Panel includes experts representing multidisciplinary specialties, including supportive care (palliative care, pain management, pastoral care, and oncology social work), anesthesiology, nursing, medical oncology, hematology oncology, internal medicine, neurology/neuro-oncology, radiotherapy/radiation oncology, psychiatry or psychology, complementary and alternative medicine, and pharmacology. The NCCN Guidelines are updated at least annually, with the most recent version at the time of writing published in June 2019 (Version 3.2019).

Recommendations in the NCCN Guidelines for Adult Cancer Pain are made based on expert panel consensus following a critical review of the best available evidence, including randomized controlled trials, observational studies, clinical trial data, and other peer reviewed data. Additionally, the NCCN Guidelines consider feedback, public comment, and evidence submission prior to the annual review of the guideline and on a rolling basis. Guidelines may be, and frequently are, reviewed and updated more often than annually. Feedback is provided during educational programs and forums. Additionally, user feedback is continually received through an online, 


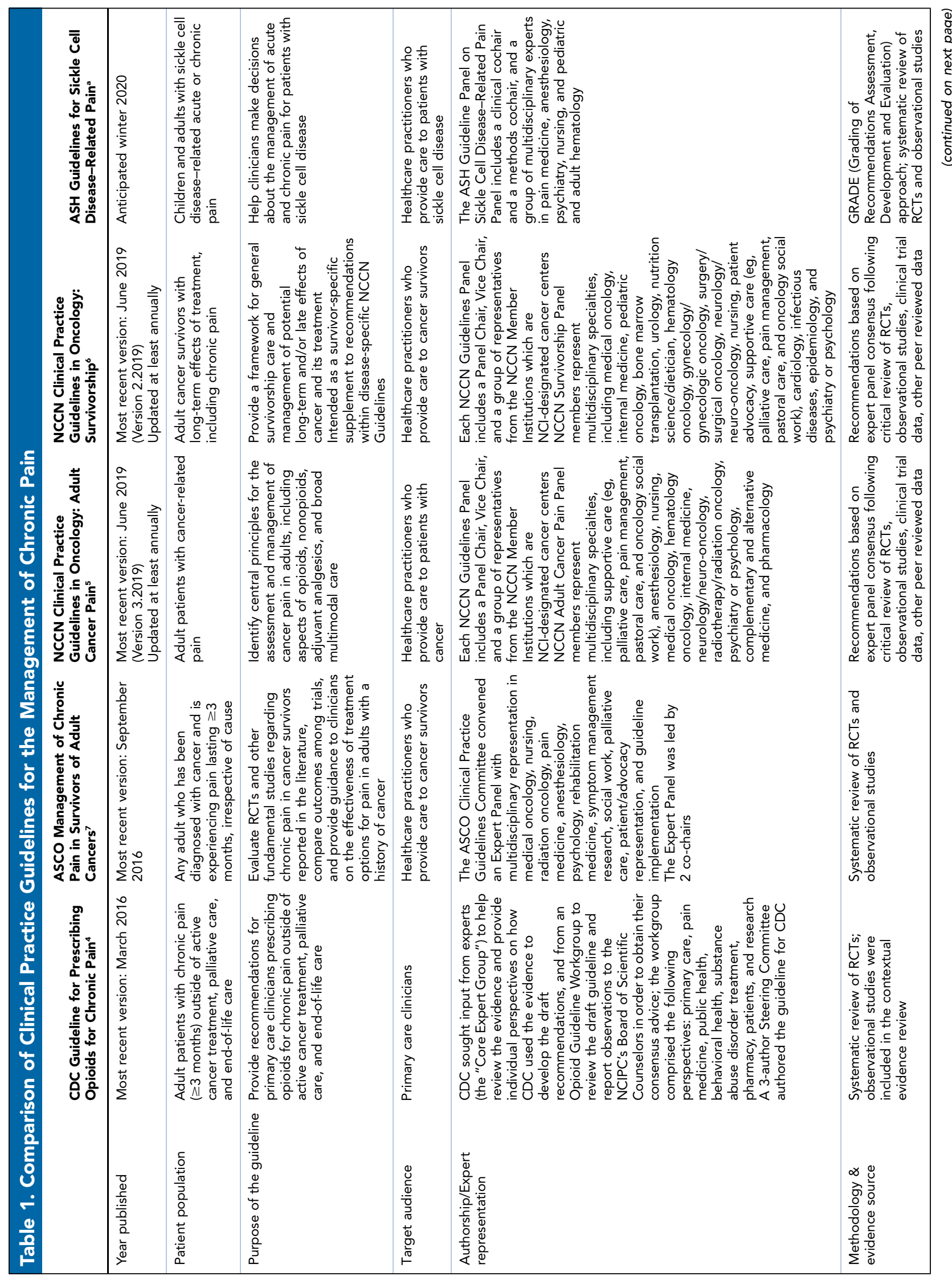




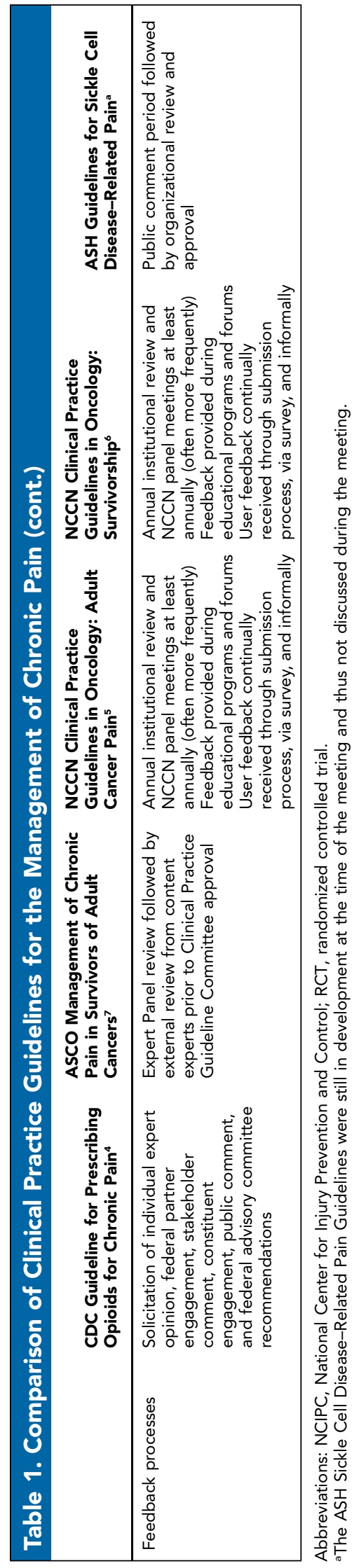

public, and transparent submission process and via a survey of guideline users, as well as informally.

\section{NCCN Guidelines for Survivorship}

The NCCN Guidelines for Survivorship provide a framework for general survivorship care and management of potential long-term and/or late effects of cancer and its treatment. ${ }^{6}$ These guidelines are intended to serve as a survivor-specific supplement to recommendations within disease-specific NCCN Guidelines. As such, the target audience of the NCCN Survivorship Guidelines is healthcare practitioners who provide care to cancer survivors, and the patient population covered by these guidelines is adult cancer survivors with long-term effects of treatment, including chronic pain. The NCCN Guidelines for Survivorship are authored by a panel of experts representing NCCN Member Institutions. The NCCN Survivorship Panel includes experts representing multidisciplinary specialties, including medical oncology, internal medicine, pediatric oncology, bone marrow transplantation, urology, nutrition science/dietician, hematology oncology, gynecology/gynecologic oncology, surgery/surgical oncology, neurology/neuro-oncology, nursing, patient advocacy, supportive care (palliative care, pain management, pastoral care, and oncology social work), cardiology, infectious diseases, epidemiology, and psychiatry or psychology. The NCCN Guidelines are updated at least annually, with the most recent version at the time of writing published in June 2019 (Version 2.2019).

Recommendations in the NCCN Guidelines for Survivorship are made based on expert panel consensus following a critical review of the best available evidence, including randomized controlled trials, observational studies, clinical trial data, and other peer reviewed data. Additionally, the NCCN Guidelines consider feedback, public comment, and evidence submission prior to the annual review of the guideline and on a rolling basis. Guidelines may be, and frequently are, reviewed and updated more often than annually. Feedback is provided during educational programs and forums. Additionally, user feedback is continually received through an online, public, and transparent submission process and via a survey of guideline users, as well as informally.

\section{ASCO Guideline on Management of Chronic Pain in} Survivors of Adult Cancers

The ASCO Clinical Practice Guideline on Management of Chronic Pain in Survivors of Adult Cancers offers evidencebased guidance on the optimum management of chronic pain in adult cancer survivors. ${ }^{7}$ In recognition of the increasing need for guidance, the ASCO Survivorship Guideline Advisory Group recommended this guideline topic as a high priority for development. Based on a 
systematic review, randomized controlled trials and other studies regarding chronic pain in cancer survivors reported in the literature are summarized, with outcomes compared, and the effectiveness of treatment options is reported. Outcomes of interest included symptom relief, pain intensity, quality of life, functional outcomes, adverse events, drug misuse or diversion, and risk assessment or mitigation. The target audience of the ASCO Management of Chronic Pain guideline is healthcare practitioners who provide care to cancer survivors, and the patient population covered by these guidelines is any adult who has been diagnosed with cancer and is experiencing pain lasting $\geq 3$ months, irrespective of etiology. The ASCO Clinical Practice Guidelines Committee convened an Expert Panel, led by 2 co-chairs, which included multidisciplinary representation in medical oncology, nursing, radiation oncology, pain medicine, anesthesiology, psychology, rehabilitation medicine, symptom management research, social work, palliative care, patient/advocacy representation, and guideline implementation. Expert Panel member review and approval was followed by Survivorship Guideline Advisory Group member review prior to external review. All ASCO guidelines are reviewed and approved by the ASCO Clinical Practice Guideline Committee prior to submission to the Journal of Clinical Oncology for consideration for publication. The guideline was published in September of 2016. The ASCO Expert Panel and guidelines staff work with co-chairs to keep abreast of any substantive updates needed in the guideline and based on formal review of the emerging literature. ASCO determines the need to update.

\section{Considerations for Pain Management of Patients With Sickle Cell Disease}

Although guidelines for the management of sickle cell disease were not a specific focus of the meeting, challenges in appropriate opioid prescribing were discussed in this patient population. The National Heart, Lung, and Blood Institute of the United States Department of Health and Human Services published guidelines for the Evidence-based Management of Sickle Cell Disease in $2014 .{ }^{11}$ These guidelines include recommendations regarding the management of chronic pain and acute pain episodes. Recommendations to treat acute pain include intervening promptly with both nonpharmacologic interventions, including heat, and pharmacologic interventions, including opioids and anti-inflammatory agents. Recommendations to treat chronic pain also include nonpharmacologic interventions, including massage, hypnosis, and muscle relaxation, and pharmacologic interventions, including opioids. When opioids are used, the guidelines encourage one primary provider of opioids and support the management of opioid adverse effects.
ASH recently published Draft Recommendations on Sickle Cell Disease-Related Pain for public comment and feedback. These new evidence-based guidelines on managing pain in sickle cell disease will provide further guidance and clarification and are anticipated for final publication in 2020.

Dr. Deepika Darbari provided a presentation of pain management needs among patients with sickle cell disease at the "Bridging the Critical Divide Among Clinical Practice Guidelines for Cancer Pain and Sickle Cell Disease" meeting. ${ }^{8}$ Dr. Darbari noted that pain for patients with sickle cell disease often begins early in life with presentation of dactylitis, a painful swelling of the hands and feet, beginning between 6 and 12 months of age and caused by abnormal sickle-shaped erythrocytes disrupting blood flow in small vessels. Following early childhood, the characteristic manifestation of sickle cell disease-related pain is acute vaso-occlusive pain crisis, which can be precipitated by temperature changes, stress, infection, dehydration, or unknown factors. Treatment of these acute pain events in childhood and adulthood involves analgesics, both opioid and nonopioid, and supportive care. There are multiple mechanisms of pain within sickle cell disease and they are not fully understood. Compounding the challenges associated with managing sickle cell disease-related pain is the wide variability of acute pain events that patients experience. Despite the significant pain, management needs, and challenges among patients with sickle cell disease, this patient population often faces more restrictive access and utilization management policies than patients with cancer-related pain. ${ }^{12}$

\section{Common Guideline Themes}

Although the identified clinical practice guidelines for pain management vary in terms of their patient population, target audience, and purpose, they do have some overlap in scope and include a number of common themes and recommendations (Table 2). The guidelines all recommend the use of nonpharmacologic therapy and nonopioid pharmacologic therapy and support the optimization of adjuvant analgesics, psychosocial support, and interventional therapies in conjunction with opioid therapy. Additionally, all of the guidelines encourage assessment of an individual's likely benefit and risk before initiating opioid treatment; development and implementation of strategies to maintain patient safety and minimize the risk of opioid misuse based on a patient's history and risk factors; and patient education regarding goals of treatment and the safe use of opioid analgesics. Across the guidelines, continuous monitoring and regular evaluations of effectiveness and necessity of opioid therapy as appropriate are encouraged. Lastly, when indicated, all of the guidelines note that opioid 


\section{Table 2. Common Themes for Pain Management Endorsed by All Guidelines}

Use of nonpharmacologic therapy and nonopioid pharmacologic therapy

Assessment of an individual's likely benefit and risk prior to initiating opioid treatment

Development and implementation of strategies to maintain patient safety and minimize the risk of opioid misuse based on patient history and risk factors

Continuous monitoring and regular evaluations of effectiveness and necessity of opioid therapy

Patient education on goals of treatment and safer use of opioid analgesics

Optimization of adjuvant analgesics, psychosocial support, and interventional therapies in conjunction with opioid therapy

Gradual opioid dose reduction, when indicated, to prevent withdrawal symptoms

dose reduction should be gradual to prevent withdrawal symptoms.

\section{Discussion of Barriers}

In addition to exploring guideline recommendations, perceived differences, and commonalities, attendees discussed common barriers to patient access to appropriate pain management tools, including opioid and nonopioid interventions. Barriers discussed included inappropriate implementation of clinical practice guidelines, insurance coverage and financial toxicity issues (ie, problems related to the cost of cancer treatment), limited market availability of appropriate formulations of opioids, and insufficient education provided to prescribers of opioids.

Attendees first discussed the inappropriate implementation and interpretation of varying clinical practice guidelines by payers that has led to improper denials and issues concerning access to care. Additionally, although all of the identified practice guidelines endorse the use of nonopioid pharmacologic and nonpharmacologic therapies, low rates of insurance coverage or limits on coverage for these pain management options often lead to significant issues regarding affordability and access to care. ${ }^{1,13,14}$ Recently, the Centers for Medicare \& Medicaid Services released the 2020 Medicare Advantage and Part D Advance Notice Part II and Draft Call Letter, which encourages Medicare Advantage organizations to consider Part $\mathrm{C}$ benefit designs for supplemental benefits that address medically approved nonopioid pain management and complementary and integrative treatments. ${ }^{15}$ Additional coverage across payers for nonopioid pain management therapies in benefit plans could help make these options more available and accessible.

Attendees also discussed the value that varying formulations of opioids, including low-dose extended-release and low-dose immediate-release formulations, which are currently unavailable on the market, could bring in terms of greater prescriber flexibility. Industry partners could consider working with providers and patient advocates to better understand pain management needs and inform the development of products to meet these needs.

There was also vigorous discussion of education gaps for prescribers of opioids. Attendees agreed that a better understanding of appropriate care for patients with pain management needs who have comorbid substance use disorders could especially help improve outcomes. Additionally, prescribers could benefit from resources on how to identify and manage misuse of opioids. Clinical practice guideline developers could also consider expanding guidance on how to appropriately taper opioid therapy.

Lastly, attendees emphasized that it could cause harm to abruptly end relationships with patients when an inappropriate substance use issue is identified. Prescribers may need additional training to appropriately transition patients with an inappropriate substance use issue, who are dependent on other services, to avoid abandoning patients in need and to ensure patients are connected to appropriate treatment services.

\section{Conclusions}

The recent JAMA Oncology article $^{3}$ appropriately calls attention to the confusion that exists among prescribers, payers, and policymakers when evaluating recommendations across multiple clinical practice guidelines for pain management. The convening of these stakeholders and presentation of each clinical practice guideline revealed that although each guideline varies in the patient population covered, target audience, methodology, and feedback processes, there is not in fact disagreement among recommendations when applied to the appropriate patient and clinical situation. Although there is some potential overlap among guidelines, further examination showed that clarification and education regarding the intent, patient population, and scope of each clinical practice guideline, rather than harmonization of guideline recommendations, could help alleviate confusion among prescribers, payers, and policymakers.

In April 2019, NCCN, ASCO, and ASH announced a key clarification received from CDC in response to a joint letter (dated February 2019) stating that the CDC guideline is not intended to deny clinically appropriate opioid therapy to any patients who experience acute or chronic pain from conditions such as cancer and sickle cell disease. ${ }^{16,17}$ Importantly, CDC clarified that the clinical practice guidelines addressing pain control 
for survivors of cancer, such as the ASCO Guideline on Management of Chronic Pain in Survivors of Adult Cancers or the NCCN Guidelines that were published and/or updated more recently than CDC's guideline, provide useful guidance on unique considerations for use of opioids to control pain in cancer survivors.

Pain management is a complex and multifactorial challenge, but there is a balance that can be struck between the benefits and harms of any pain management strategy, including those involving opioids. Meeting attendees acknowledged that there are select populations of patients, including those with sickle cell disease, those undergoing active cancer treatment, and some survivors of cancer with no evidence of active disease, for whom unique considerations would change the balance of benefits and risks regarding opioid use for pain management. The hope is that publication of this paper represents a first step toward providing clarity regarding perceived guideline variance. Clinical practice guidelines offer an important tool to inform evidence-based state, federal, and coverage policies addressing pain management for individuals with sickle cell disease, patients undergoing active cancer treatment, and cancer survivors. As our nation moves forward with efforts to address the opioid crisis and balance patient pain management needs, clinical practice guidelines can serve as a resource for policymakers and payers to help inform policy, coverage determinations, and treatment decisions.

\section{Submitted October 25, 2019; accepted for publication November 12, 2019.}

Publication: This is a dual publication with JCO Oncology Practice.

Disclosures: Ms. Schatz has disclosed that her spouse holds stock in TransEnterix, Editas Medicine, Amgen, Adamas Pharmaceuticals, UnitedHealth, Intuitive Surgical, and Akers Biosciences. Dr. Darbari has disclosed that she is a scientific advisor for Novartis, and served on the advisory board for Global Blood Therapeutics. Dr. Bruera has disclosed that he receives research support from Helsinn Health. The remaining authors have disclosed that they have no financial interests, arrangements, affiliations, or commercial interests with the manufacturers of any products discussed in this article or their competitors.

Disclaimer: The views expressed in this article are those of the authors and do not necessarily represent the official position of the National Comprehensive Cancer Network, American Society of Clinical Oncology, American Society of Hematology, or Centers for Disease Control and Prevention.

Correspondence: Alyssa A. Schatz, MSW, National Comprehensive Cancer Network, 3025 Chemical Road, Suite 100, Plymouth Meeting, PA 19462. Email: Schatz@nccn.org

\section{References}

1. Page R, Blanchard E. Opioids and cancer pain: patients' needs and access challenges. J Oncol Pract 2019;15:229-231.

2. Sinha CB, Bakshi N, Ross D, et al. Management of chronic pain in adults living with sickle cell disease in the era of the opioid epidemic: a qualitative study. JAMA Netw Open 2019;2:e194410.

3. Meghani $\mathrm{SH}$, Vapiwala N. Bridging the critical divide in pain management guidelines from the CDC, NCCN, and ASCO for cancer survivors. JAMA Oncol 2018;4:1323-1324.

4. Dowell D, Haegerich TM, Chou R. CDC guideline for prescribing opioids for chronic pain - United States, 2016. MMWR Recomm Rep 2016;65: $1-49$.

5. Swarm RA, Paice JA, Anghelescu DL, et al. NCCN Clinical Practice Guidelines in Oncology: Adult Cancer Pain, Version 3.2019. Accessed February 14, 2020. To view the most recent version, visit NCCN.org.

6. Denlinger CS, Sanft T, Armenian S, et al. NCCN Clinical Practice Guidelines in Oncology: Survivorship, Version 2.2019. Accessed February 14, 2020. To view the most recent version, visit NCCN.org

7. Paice JA, Portenoy R, Lacchetti C, et al. Management of chronic pain in survivors of adult cancers: American Society of Clinical Oncology clinical practice guideline. J Clin Oncol 2016;34:3325-3345.

8. Darbari D. Presentation at the Bridging the Critical Divide Among Clinical Practice Guidelines for Cancer Pain and Sickle Cell Disease meeting; November 8th, 2018; Washington, DC.

9. Guyatt $G H$, Oxman $A D$, Vist GE, et al. GRADE: an emerging consensus on rating quality of evidence and strength of recommendations. BMJ 2008; 336:924-926.

10. Dowell D, Haegerich TM, Chou R. CDC guideline for prescribing opioids for chronic pain - United States, 2016. JAMA 2016;315:1624-1645.

11. National Heart, Lung, and Blood Institute. Evidence-based management of sickle cell disease. Expert panel report, Accessed February 25, 2019. Available at: http://www.nhlbi.nih.gov/sites/www.nhlbi.nih.gov/files/ sickle-cell-disease-report.pdf

12. National Council of State Legislatures. Prescribing policies: States confront opioid overdose epidemic. Table: State prescribing legislation. Accessed August 18, 2019. Available at: http://www.ncsl.org/research/ health/prescribing-policies-states-confront-opioid-overdose-epidemic. aspx

13. Lin $\mathrm{DH}$, Jones $\mathrm{CM}$, Compton WM, et al. Prescription drug coverage for treatment of low back pain among US Medicaid, Medicare Advantage, and commercial insurers. JAMA Netw Open 2018;1:e180235.

14. Heyward J, Jones CM, Compton WM, et al. Coverage of nonpharmacologic treatments for low back Pain among US public and private insurers. JAMA Netw Open 2018;1:e183044.

15. Center for Medicare and Medicaid Services. Advance notice of methodological changes for calendar year (CY) 2020 for Medicare Advantage (MA) capitation rates, part $C$ and part D payment policies and 2020 draft call letter. Accessed February 22, 2019. Available at: https://www.cms. gov/Medicare/Health-Plans/MedicareAdvtgSpecRateStats/Downloads/ Advance2020Part2.pdf

16. National Comprehensive Cancer Network. CDC issues key clarification on guideline for prescribing opioids for chronic pain. Accessed August 2,3 2019. Available at: https://www.nccn.org/about/news/newsinfo.aspx? NewsID $=1490$

17. American Society of Clinical Oncology. CDC issues key clarification on guideline for prescribing opioids for chronic pain. Accessed August 2,3 2019. Available at: https://www.ascopost.com/issues/may-10-2019/cdcissues-clarification-on-guideline-for-prescribing-opioids-for-chronic-pain/ 